\title{
Author Correction: Gain-of-function variants in SYK cause immune dysregulation and systemic inflammation in humans and mice
}

Lin Wang (iD, Dominik Aschenbrenner (iD, Zhiyang Zeng, Xiya Cao, Daniel Mayr, Meera Mehta, Melania Capitani, Neil Warner (D, Jie Pan, Liren Wang, Qi Li, Tao Zuo, Sarit Cohen-Kedar, Jiawei Lu, Rico Chandra Ardy, Daniel J. Mulder (D, Dilan Dissanayake, Kaiyue Peng, Zhiheng Huang, Xiaoqin Li, Yuesheng Wang, Xiaobing Wang, Shuchao Li, Samuel Bullers, Anís N. Gammage iD, Klaus Warnatz, Ana-Iris Schiefer, Gergely Krivan, Vera Goda, Walter H. A. Kahr (D, Mathieu Lemaire, Genomics England Research Consortium*, Chien-Yi Lu, Iram Siddiqui, Michael G. Surette (D), Daniel Kotlarz, Karin R. Engelhardt, Helen R. Griffin, Robert Rottapel, Hélène Decaluwe, Ronald M. Laxer, Michele Proietti, Sophie Hambleton (D), Suzanne Elcombe, Cong-Hui Guo, Bodo Grimbacher, Iris Dotan, Siew C. Ng (iD, Spencer A. Freeman, Scott B. Snapper (D), Christoph Klein (D), Kaan Boztug, Ying Huang (D), Dali Li (D), Holm H. Uhlig and Aleixo M. Muise (iD)

Correction to: Nature Genetics https://doi.org/10.1038/s41588-021-00803-4, published online 29 March 2021.

In the version of this article initially published, there was a numbering error in the affiliation for Christoph Klein. The author's proper affiliation is 30, Department of Pediatrics, Dr. von Hauner Children's Hospital, University Hospital, Ludwig Maximilian University Munich, Munich, Germany, and not Newcastle University, UK. The change has been made to the online version of the article.

${ }^{\star} \mathrm{A}$ list of authors and their affiliations appears online.

Published online: 5 January 2022

https://doi.org/10.1038/s41588-021-00998-6

๑) The Author(s), under exclusive licence to Springer Nature America, Inc. 2022

\section{Author Correction: Mutations in PYCR1 cause cutis laxa with progeroid features}

Bruno Reversade, Nathalie Escande-Beillard, Aikaterini Dimopoulou, Björn Fischer, Serene C. Chng, Yun Li, Mohammad Shboul, Puay-Yoke Tham, Hülya Kayserili, Lihadh Al-Gazali, Monzer Shahwan, Francesco Brancati, Hane Lee, Brian D. O'Connor, Mareen Schmidt-von Kegler, Barry Merriman, Stanley F. Nelson, Amira Masri, Fawaz Alkazaleh, Deanna Guerra, Paola Ferrari, Arti Nanda, Anna Rajab, David Markie, Mary Gray, John Nelson, Arthur Grix, Annemarie Sommer, Ravi Savarirayan, Andreas R. Janecke, Elisabeth Steichen, David Sillence, Ingrid Haußer, Birgit Budde, Gudrun Nürnberg, Peter Nürnberg, Petra Seemann, Désirée Kunkel, Giovanna Zambruno, Bruno Dallapiccola, Markus Schuelke, Stephen Robertson, Hanan Hamamy, Bernd Wollnik, Lionel Van Maldergem, Stefan Mundlos and Uwe Kornak

Correction to: Nature Genetics https://doi.org/10.1038/ng.413, published online 2 August 2009.

In the version of this article initially published, there was an omission in the Acknowledgements section for support to Hülya Kayserili. The Acknowledgements should have ended with the text "and a grant from TUBITAK SBAG-108S418 to H.K."

Published online: 21 January 2022

https://doi.org/10.1038/s41588-022-01013-2

( $)$ The Author(s), under exclusive licence to Springer Nature America, Inc. 2022 